\title{
Lipschitz conditions on the modulus of a harmonic function
}

Miroslav Pavlović

\begin{abstract}
It is proved that if $u$ is a real valued function harmonic in the open unit ball $\mathbb{B}_{N} \subset \mathbb{R}^{N}$ and continuous on the closed ball, then the following conditions are equivalent, for $0<\alpha<1$ :

- $|u(x)-u(w)| \leq C|x-w|^{\alpha}, \quad x, w \in \mathbb{B}_{N}$;

- ||$u(y)|-| u(\zeta)|| \leq C|y-\zeta|^{\alpha}, \quad y, \zeta \in \partial \mathbb{B}_{N}$;

- ||$u(y)|-| u(r y)|| \leq C(1-r)^{\alpha}, \quad y \in \partial \mathbb{B}_{N}, 0<r<1$.
\end{abstract}

The Lipschitz condition on $|u|^{p}$ is also considered.

\section{Introduction}

Let $S$ be a subset of the $N$-dimensional euclidean space $\mathbb{R}^{N}$, and let $\omega$ be a majorant, i.e., a continuous increasing function on $[0, \infty)$ such that $\omega(0)=0$ and $\omega(t) / t$ is non-increasing for $t>0$.

For a real or complex valued function $f$ on $S$, we write $f \in \Lambda_{\omega}(S)$ if there is a constant $C<\infty$ such that

$$
|f(x)-f(w)| \leq C \omega(|x-w|), \quad x, w \in S .
$$

If $\omega(t)=t^{\alpha}, 0<\alpha \leq 1$, then we write $\Lambda_{\omega}(S)=\Lambda^{\alpha}(S)$.

Let $\mathbb{B}_{N}$ denote the open unit ball of $\mathbb{R}^{N}$. For a function $f$ defined and continuous on the closure $\overline{\mathbb{B}}_{N}$ of $\mathbb{B}_{N}$ consider the following conditions:

(A) $f \in \Lambda_{\omega}\left(\mathbb{B}_{N}\right)$;

(B) $f \in \Lambda_{\omega}\left(\partial \mathbb{B}_{N}\right)$;

(C) There is a constant $C<\infty$ such that $|f(y)-f(r y)| \leq C \omega(1-r)$, for $y \in \partial \mathbb{B}_{N}, 0<r<1$ 
(A1) $|f| \in \Lambda_{\omega}\left(\mathbb{B}_{N}\right)$

(B1) $|f| \in \Lambda_{\omega}\left(\partial \mathbb{B}_{N}\right)$;

(C1) There is a constant $C<\infty$ such that ||$f(y)|-| f(r y)|| \leq C \omega(1-r)$, for $y \in \partial \mathbb{B}_{N}, 0<r<1$.

Clearly there hold the implications: $(\mathrm{A}) \Longrightarrow(\mathrm{A} 1),(\mathrm{B}) \Longrightarrow(\mathrm{B} 1)$ and $(\mathrm{C}) \Longrightarrow(\mathrm{C} 1)$. In a series of papers $[4,5,6,7]$, Dyakonov considered the implications $(\mathrm{A} 1) \Longrightarrow(\mathrm{A})$ and $(\mathrm{B} 1+\mathrm{C} 1) \Longrightarrow(\mathrm{A})$ for various classes of functions (defined on more general domains). In [4], he proved the validity of these implications in the case where $f$ is holomorphic in the unit disk $\mathbb{D}=\mathbb{B}_{2}$ of the complex plane $\mathbb{C}=\mathbb{R}^{2}$; the majorant was assumed to satisfy the condition

$$
\int_{0}^{x} \frac{\omega(t)}{t} d t+x \int_{x}^{\infty} \frac{\omega(t)}{t^{2}} d t \leq C \omega(x), \quad x>0 \quad(C=\text { const }) .
$$

Following Dyakonov we call such a majorant regular. In [5], another interesting condition is considered, namely:

(D) There is a constant $C<\infty$ such that $\mathcal{P}[|f|](x)-|f(x)| \leq C \omega(1-|x|)$, for $x \in \mathbb{B}_{N}$,

where $\mathcal{P}[|f|]$ denotes the Poisson integral of the boundary function,

$$
\mathcal{P}[|f|](x)=\int_{\partial \mathbb{B}_{N}} \frac{1-|x|^{2}}{|x-y|^{N}}|f(y)| d \sigma(y),
$$

and $d \sigma$ is the normalized surface measure on the unit sphere. It is proved in $[4]$ that $(\mathrm{B} 1+\mathrm{D})$ is equivalent to $(\mathrm{A})$.

The following condition together with Lemma A below is often used when verifying $(\mathrm{A})$ :

(E) $f \in \Lambda_{1}(K)$ for every compact subset $K$ of $\mathbb{B}_{N}$, and there is a constant $C$ such that

$$
|\nabla f(x)| \leq C \frac{\omega(1-|x|)}{1-|x|} \quad \text { for almost all } x \in \mathbb{B}_{N}
$$

Lemma A. Let $\omega$ be fast, i.e.,

$$
\int_{0}^{x} \frac{\omega(t)}{t} d t \leq C \omega(x), \quad x>0
$$

If $f$ satisfies $(\mathrm{E})$, then $f \in \Lambda_{\omega}\left(\mathbb{B}_{N}\right)$.

(See Lemma 3 below.) 
We collect Dyakonov's results together with some classical facts in the following way:

Theorem A. Let $\omega$ be a regular majorant, and let $f$ be a function holomorphic in $\mathbb{B}_{2}$. Then there hold the relations:

$$
(\mathrm{A}) \Longleftrightarrow(\mathrm{B}) \Longleftrightarrow(\mathrm{C}) \Longleftrightarrow(\mathrm{A} 1) \Longleftrightarrow(\mathrm{B} 1+\mathrm{C} 1) \Longleftrightarrow(\mathrm{B} 1+\mathrm{D}) \text {. }
$$

Part of (1.2), namely

$$
(\mathrm{A}) \Longleftrightarrow(\mathrm{B}) \Longleftrightarrow(\mathrm{C}) \Longleftrightarrow(\mathrm{A} 1)
$$

holds under the (weaker) hypothesis $(1.1)$ ( $f$ holomorphic); for $(\mathrm{A}) \Longleftrightarrow(\mathrm{A} 1)$, see [18, Theorems 10.2.1 and 10.2.2] and [6]; for $(A) \Longleftrightarrow(B) \Longleftrightarrow(C)$, see, e.g., $[13,14]$ and Lemma 5 below. A simple proof of the equivalences $(\mathrm{A} 1) \Longleftrightarrow(\mathrm{B} 1+\mathrm{C} 1) \Longleftrightarrow(\mathrm{B} 1+\mathrm{D})$ is given in [17] (see also [2]).

It is perhaps worthwhile to note that the proofs given in [4] and [17] show that condition ( $\mathrm{C} 1)$ in Theorem A can be replaced by a weaker one, namely:

(C2) There is a constant $C<\infty$ such that $|f(y)|-|f(r y)| \leq C \omega(1-r)$, for $y \in \partial \mathbb{B}_{N}, 0<r<1$.

(See [18, Theorem 10.2.3].)

In this note we are mainly concerned with the case where $f$ is real valued and harmonic in $\mathbb{B}_{N}$, and we prove the validity of the relations

$$
(\mathrm{A}) \Longleftrightarrow(\mathrm{B}) \Longleftrightarrow(\mathrm{C}) \Longleftrightarrow(\mathrm{A} 1) \Longleftrightarrow(\mathrm{B} 1) \Longleftrightarrow(\mathrm{C} 1)
$$

under the hypothesis that the majorant is regular (Theorems 1 and 2); we do not know whether $(\mathrm{C} 1)$ can be replaced by $(\mathrm{C} 2)$.

Concerning condition $(\mathrm{B} 1+\mathrm{D})$ ( $f$ real valued), note the following: (a) (D) is satisfied by any positive harmonic function; (b) it can be shown that (A) implies (D); (c) and since (B1) implies (A), we see that (B1) implies (D). Thus it seems that considering condition (D) in the real valued context makes no sense.

In the case where $f$ is complex valued and harmonic, we can only prove that condition (A1) implies $f \in \Lambda_{\sqrt{\omega}}\left(\mathbb{B}_{N}\right)$ (see Theorem 3 below and $[7$, Theorem 3.2]).

Finally, it may be of some interest to study the implication $(\mathrm{A}) \Longrightarrow(\mathrm{E})$. It is well known and easy to see that this implication holds, for arbitrary majorants, in the class of complex valued harmonic functions, and, in particular, in the class of holomorphic functions. Here we consider some nonlinear classes, such as $\left\{|u|^{p}: u\right.$ real valued harmonic $\}, p>1$ (see Theorem 4). 


\section{Statement of results}

Throughout the note we use the notation

$$
M(r, u)=M_{\infty}(r, u)=\max _{y \in \partial \mathbb{B}_{N}}|u(r y)| .
$$

We denote by $H\left(\mathbb{B}_{N}, \mathbb{R}\right)$, respectively $H\left(\mathbb{B}_{N}, \mathbb{C}\right)$, the class of all real valued, respectively complex valued, functions harmonic in $\mathbb{B}_{N}$. The subclass of $H\left(\mathbb{B}_{N}, \mathbb{R}\right)$, respectively $H\left(\mathbb{B}_{N}, \mathbb{C}\right)$, consisting of functions that are continuous on $\overline{\mathbb{B}}_{N}=\mathbb{B}_{N} \cup \partial \mathbb{B}_{N}$ will be denoted by $H\left(\overline{\mathbb{B}}_{N}, \mathbb{R}\right)$, respectively $H\left(\overline{\mathbb{B}}_{N}, \mathbb{C}\right)$.

As in [5], we call a majorant $\omega$ fast if it satisfies (1.1). And, $\omega$ is called slow if it satisfies

$$
\int_{x}^{\infty} \frac{\omega(t)}{t^{2}} d t \leq C \frac{\omega(x)}{x}, \quad x>0 .
$$

Hence, $\omega$ is regular if and only if it is both fast and slow. It turns out that these notions can be described in a very simple way.

Proposition 1. [19, Proposition 2] A majorant $\omega$ is fast, respectively slow, if and only if

$$
\text { there are constants } \alpha>0 \text { and } C>0 \text { such that }
$$

$$
\omega(\lambda t) \leq C \lambda^{\alpha} \omega(t) \quad(t>0,0<\lambda<1)
$$

respectively

$$
\text { there are constants } \beta<1 \text { and } C>0 \text { such that }
$$

$$
\omega(\lambda t) \leq C \lambda^{\beta} \omega(t) \quad(t>0, \lambda>1) .
$$

Instead of (2.1), respectively (2.2), we can say " $\omega(t) / t^{\alpha}$ is almost increasing", respectively " $\omega(t) / t^{\beta}$ is almost decreasing".

Our first result is a direct consequence of a simple but useful observation of Dyakonov (Lemma 1) and the fact that the Poisson integral of a function of the class $\Lambda_{\omega}\left(\partial \mathbb{B}_{N}\right)$ ( $\omega$ slow) belongs to $\Lambda_{\omega}\left(\mathbb{B}_{N}\right)$ (Lemma 2 ).

Theorem 1. If $\omega$ is slow, $u \in H\left(\overline{\mathbb{B}}_{N}, \mathbb{R}\right)$ and $|u| \in \Lambda_{\omega}\left(\partial \mathbb{B}_{N}\right)$, then $u \in \Lambda_{\omega}\left(\mathbb{B}_{N}\right)$.

Lemma 1. [7, Lemma 3.1] Let $S=\partial \mathbb{B}_{N}$ or $S=\mathbb{B}_{N}$. If $\varphi: S \mapsto \mathbb{R}$ is a continuous function such that $|\varphi| \in \Lambda_{\omega}(S)$, then $\varphi \in \Lambda_{\omega}(S)$.

Lemma 2. [5, Lemma 1] If $U \in \Lambda_{\omega}\left(\partial \mathbb{B}_{N}\right) \cap H\left(\overline{\mathbb{B}}_{N}, \mathbb{C}\right)$, where $\omega$ is a slow majorant, then $U \in \Lambda_{\omega}\left(\mathbb{B}_{N}\right)$.

In [5], this lemma was stated in the case of the unit ball of $\mathbb{C}^{N}$. For the proof see Lemma 3.2 in [12, pp. 268-269] and [4, Lemma 4]. 
Theorem 2. If $\omega$ is regular, and $u \in H\left(\overline{\mathbb{B}}_{N}, \mathbb{R}\right)$ satisfies the condition

$$
|| u(y)|-| u(r y)|| \leq C \omega(1-r), \quad 0<r<1, y \in \partial \mathbb{B}_{N},
$$

where $C$ is independent of $r, y$, then $u \in \Lambda_{\omega}\left(\mathbb{B}_{N}\right)$.

As follows from Lemma 1 , the implication $(\mathrm{A} 1) \Longrightarrow(\mathrm{A})$ holds under the hypothesis that $f$ is real valued and continuous; in the case where $f$ is complex valued and continuous, and $0<\alpha \leq 1$, Dyakonov [7] proved the validity of the implication

$$
|f| \in \Lambda^{\alpha}(\mathbb{D}) \text { and } \operatorname{Re} f \in \Lambda^{\alpha}(\mathbb{D}) \Longrightarrow f \in \Lambda^{\alpha / 2}(\mathbb{D}) .
$$

The conclusion $f \in \Lambda^{\alpha / 2}(\mathbb{D})$ is best possible even in the class of all harmonic functions (see [7, Theorem 3.2]). We will prove that if $f$ is harmonic, then (2.4) remains valid if we remove the condition $\operatorname{Re} f \in \Lambda^{\alpha}(\mathbb{D})$ :

Theorem 3. Let $\omega$ be a fast majorant. If $f \in H\left(\mathbb{B}_{N}, \mathbb{C}\right)$ and $|f|^{2} \in \Lambda_{\omega}\left(\mathbb{B}_{N}\right)$, and in particular if $|f| \in \Lambda_{\omega}\left(\mathbb{B}_{N}\right)$, then $f \in \Lambda_{\sqrt{\omega}}\left(\mathbb{B}_{N}\right)$.

Theorem 4. Let $\omega$ be an arbitrary majorant, and let $g=|f|^{p}$, where $f$ and $p$ satisfy one of the following conditions:

(a) $f$ is holomorphic in $\mathbb{B}_{N}(N=2), p>1$;

(b) $f \in H\left(\mathbb{B}_{N}, \mathbb{R}\right), p>1$;

(c) $f \in H\left(\mathbb{B}_{N}, \mathbb{C}\right), p \geq 2$.

If $g \in \Lambda_{\omega}\left(\mathbb{B}_{N}\right)$, then there is a constant $C$ such that

$$
|\nabla g(x)| \leq C \frac{\omega(1-|x|)}{1-|x|}, \quad x \in \mathbb{B}_{N} .
$$

Because of the formula $|\nabla| f||=\left|f^{\prime}\right|$ and Lemma A, we get:

Corollary 1. Let $\omega$ be a fast majorant, and let $f$ be a function holomorphic in $\mathbb{D}$, and $p>1$. Then $|f|^{p} \in \Lambda_{\omega}(\mathbb{D})$ if and only if

$$
|f(z)|^{p-1}\left|f^{\prime}(z)\right| \leq C \frac{\omega(1-|z|)}{1-|z|}, \quad z \in \mathbb{D} .
$$

In the case $p=1$ this corollary is essentially equal to Dyakonov's theorem. If $p$ is an integer, then we can apply Dyakonov's theorem to the holomorphic function $f^{p}$.

In the case of real valued harmonic functions we have:

Corollary 2. Let $\omega$ be a fast majorant, and let $u$ be a real valued function harmonic in $\mathbb{B}_{N}$, and $p>1$. Then $|u|^{p} \in \Lambda_{\omega}\left(\mathbb{B}_{N}\right)$ if and only if

$$
|u(x)|^{p-1}|\nabla u(x)| \leq C \frac{\omega(1-|x|)}{1-|x|}, \quad x \in \mathbb{B}_{N} .
$$




\section{Proof of Theorem 2}

Theorem 2 is a consequence of the following theorem and the definition of a regular majorant.

Theorem 5. Let $\omega$ be a majorant satisfying the (Dini) condition:

$$
\int_{0}^{1} \frac{\omega(t)}{t} d t<\infty
$$

If $u \in H\left(\overline{\mathbb{B}}_{N}, \mathbb{R}\right)$ satisfies condition (2.3), where $C$ is independent of $r, y$, then $u \in \Lambda_{\omega_{3}}\left(\mathbb{B}_{N}\right)$, where

$$
\omega_{3}(x)=\int_{0}^{x} \frac{\omega(t)}{t} d t+x \int_{x}^{\infty} \frac{\omega(t)}{t^{2}} d t, \quad x>0 .
$$

Before the proof some comments are in order. Let

$$
\begin{aligned}
& \omega_{1}(x)=\int_{0}^{x} \frac{\omega(t)}{t} d t=\int_{0}^{1} \frac{\omega(x t)}{t} \\
& \omega_{2}(x)=x \int_{x}^{\infty} \frac{\omega(t)}{t^{2}} d t=\int_{1}^{\infty} \frac{\omega(x t)}{t^{2}} d t .
\end{aligned}
$$

It is clear that these functions are majorants provided they are finite. The function $\omega_{1}$ is finite by hypothesis. The function $\omega_{2}$ need not be finite (e.g., take $\omega(t)=t$ ) but since the definition of $\Lambda_{\omega}$ is independent of the values of $\omega(t)$ for $t \geq 2$, we can put, for example, $\omega(t)=\omega(2)$, for $t \geq 2$. In this way $\omega$ remains a majorant and $\omega_{2}$ becomes finite. Thus $\omega_{3}$ is a majorant as a sum of two majorants. But the following formula is more important:

$$
\omega_{3}(x)=\int_{0}^{x} \frac{\omega_{2}(t)}{t} d t, \quad x>0
$$

For the proof of Theorem 5 we need a few lemmas. The first lemma provides a sufficient condition for a $C^{1}$-function to be in $\Lambda_{\omega}\left(\mathbb{B}_{N}\right)$; for a proof see, for example, [20, Lemma 6.4.8] (the case $\omega(t)=t^{\alpha}$ ) and [18, Lemma 10.1.6].

Lemma 3. If a complex valued function $f$ satisfies condition (E), where $\omega$ satisfies the Dini condition, then $f$ is in $\Lambda_{\omega_{1}}\left(\mathbb{B}_{N}\right)$, and in particular $f$ has a continuous extension to the closed ball.

For a function $U=U_{1}+i U_{2}$, we write

$$
|\nabla U|=\sqrt{\left|\nabla U_{1}\right|^{2}+\left|\nabla U_{2}\right|^{2}}
$$

The following fact is well known, and follows from the inequality

$$
M(s,|\nabla U|) \leq C(s-\rho)^{-1} M(\rho, U), \quad 0<\rho<s<1 .
$$


Lemma 4. If $\omega$ is an arbitrary majorant, and if $U \in H\left(\mathbb{B}_{N}, \mathbb{C}\right)$ is such that

$$
M(r, U) \leq \frac{\omega(1-r)}{1-r}
$$

then

where $C$ is independent of $U$.

$$
M(r,|\nabla U|) \leq C \frac{\omega(1-r)}{(1-r)^{2}},
$$

Let $u^{\prime}$ denote the radial derivative of $u$ :

$$
u^{\prime}(r y)=\frac{\partial u}{\partial r}(r y)
$$

Lemma 5. Let $\omega$ be an arbitrary majorant. If $u \in H\left(\mathbb{B}_{N}, \mathbb{C}\right)$ satisfies

$$
|u(\rho y)-u(r y)| \leq \omega(1-r), \quad 0<r<\rho<1,|y|=1,
$$

then

$$
\left|u^{\prime}(r y)\right| \leq C \frac{\omega(1-r)}{1-r}, \quad 1 / 2<r<1,|y|=1,
$$

where $C$ is independent of $u$.

Proof. We can assume that $u$ is harmonic in a neighborhood of the closed unit ball. Then, in the general case, we apply the result to the functions $x \mapsto u(\lambda x), 0<\lambda<1$. Let

$$
A=\sup _{1 / 2<r<1} \frac{1-r}{\omega(1-r)} M\left(r, u^{\prime}\right)
$$

where

$$
M\left(r, u^{\prime}\right)=\sup _{y \in \partial \mathbb{B}_{N}}\left|u^{\prime}(r y)\right| .
$$

We start from the (Taylor) formula

$$
u(\rho y)-u(r y)=u^{\prime}(r y)(\rho-r)+\int_{r}^{\rho}(\rho-t) u^{\prime \prime}(t y) d t .
$$

Hence

$$
M\left(r, u^{\prime}\right)(\rho-r) \leq \omega(1-r)+\int_{r}^{\rho}(\rho-t) M\left(t, u^{\prime \prime}\right) d t .
$$

The function $r^{2} u^{\prime \prime}$ is harmonic, whence $r^{2} M\left(r, u^{\prime \prime}\right)$ increases with $r$, so we have

$$
M\left(r, u^{\prime}\right)(\rho-r) \leq \omega(1-r)+4 \rho^{2} \int_{r}^{\rho}(\rho-t) M\left(\rho, u^{\prime \prime}\right) d t, \quad \rho>r>1 / 2,
$$

whence

$$
M\left(r, u^{\prime}\right)(\rho-r) \leq \omega(1-r)+2(\rho-r)^{2} M\left(\rho, u^{\prime \prime}\right), \quad \rho>r>1 / 2 .
$$


On the other hand, an application of (3.4) to the harmonic function $U(r y)=r u^{\prime}(r y)$ gives

$$
M\left(\rho, u^{\prime \prime}\right) \leq K(s-\rho)^{-1} M\left(s, u^{\prime}\right), \quad s>\rho>1 / 2,
$$

where $K$ is an constant. Then, putting $\rho=r+\delta$ and $s=\rho+\varepsilon$, we get

$$
\begin{aligned}
M\left(r, u^{\prime}\right) & \leq \frac{\omega(1-r)}{\delta}+2 K \frac{\delta}{\varepsilon} M\left(r+\delta+\varepsilon, u^{\prime}\right) \\
& \leq \frac{\omega(1-r)}{\delta}+2 K \frac{\delta}{\varepsilon} A \frac{\omega(1-r-\delta-\varepsilon)}{1-r-\delta-\varepsilon} .
\end{aligned}
$$

Take

$$
\delta=a(1-r), \quad \varepsilon=b(1-r), \quad \text { where } a>0, b>0, a+b<1,
$$

to get

$$
M\left(r, u^{\prime}\right) \leq \frac{\omega(1-r)}{a(1-r)}+2 K \frac{a}{b} A \frac{\omega(1-r)}{(1-a-b)(1-r)} .
$$

Hence, by (2.1),

$$
M\left(r, u^{\prime}\right) \leq \frac{\omega(1-r)}{a(1-r)}+2 K(a / b) C(1-a-b)^{-1} A \frac{\omega(1-r)}{1-r} .
$$

Now we choose $a$ and $b$ so that

$$
2 K(a / b) C(1-a-b)^{-1}<1 / 2 .
$$

It follows that

$$
\frac{1-r}{\omega(1-r)} M\left(r, u^{\prime}\right) \leq \frac{1}{a}+A / 2, \quad r>1 / 2
$$

Hence, taking supremum on the left side, we get

$$
A \leq \frac{1}{a}+A / 2
$$

and finally $A \leq 2 / a$, which was to be proved.

It is a familiar fact that the radial derivative controls the gradient (see, e.g., [21]); we state it in the following form:

Lemma 6. If $\omega$ is a majorant, and

$$
\left|u^{\prime}(r y)\right| \leq \frac{\omega(1-r)}{1-r}, \quad 1 / 2<r<1,|y|=1,
$$

where $u \in H\left(\mathbb{B}_{N}, \mathbb{C}\right)$, then

$$
|\nabla u(r y)| \leq C \frac{\omega_{2}(1-r)}{1-r}, \quad 0<r<1,|y|=1,
$$

where $\omega_{2}$ is defined by (3.2). 
Proof. We can suppose that $u$ is real valued. Consider the (harmonic) functions

$$
\begin{aligned}
& R u(r y)=r u^{\prime}(r y)=\sum_{i=1}^{N} x_{i} D_{i} u(x), \quad x=r y,|y|=1, \\
& T_{i j} u(x)=x_{j} D_{i} u(x)-x_{i} D_{j} u(x), \quad x=\left(x_{1}, \ldots, x_{N}\right) \in \mathbb{B}_{N},
\end{aligned}
$$

where $D_{i} u=\frac{\partial u}{\partial x_{i}}$.

It follows from (3.5) and the maximum principle that

$$
M(r, R u) \leq \frac{\omega(1-r)}{1-r}, \quad 0<r<1
$$

From this, by Lemma 4, we get

$$
M\left(r, T_{i j} R u\right) \leq C r \frac{\omega(1-r)}{(1-r)^{2}}
$$

which can be written as

$$
M\left(r, R T_{i j} u\right) \leq C r \frac{\omega(1-r)}{(1-r)^{2}},
$$

because $R T_{i j}=T_{i j} R$. Hence

$$
M\left(r,\left(T_{i j} u\right)^{\prime}\right) \leq C \frac{\omega(1-r)}{(1-r)^{2}}
$$

and hence, by integration,

$$
M\left(r, T_{i j} u\right) \leq C \int_{1-r}^{1} \frac{\omega(t)}{t^{2}} d t \leq C \frac{\omega_{2}(1-r)}{1-r} .
$$

Now we use the identity

$$
(R u)^{2}+\frac{1}{2} \sum_{i, j=1}^{N}\left(T_{i j} u\right)^{2}=|x|^{2}|\nabla u|^{2}
$$

to deduce from (3.7) and (3.8) that

$$
M(r,|\nabla u|) \leq C_{1} \frac{\omega_{2}(1-r)}{1-r}, \quad 1 / 2<r<1 .
$$

Finally, applying the maximum principle to the subharmonic function $|\nabla u|$, we get the desired result.

The following lemma is similar to Lemma 3.1 of [7] (Lemma 1 above). 
Lemma 7. If a real valued continuous function $u$ on $\overline{\mathbb{B}}_{N}$ satisfies $(2.3)$, then

$$
|u(\rho y)-u(r y)| \leq 4 C \omega(1-r), \quad 0<r<\rho<1 .
$$

Proof. Let $y \in \partial \mathbb{B}_{N}$. Assume that $u(\rho y) u(r y) \geq 0$. Then

$$
\begin{aligned}
|u(\rho y)-u(r y)| & =|| u(\rho y)|-| u(r y)|| \\
& \leq|| u(r y)|-| u(y)||+|| u(\rho y)|-| u(y)|| \\
& \leq C \omega(1-\rho)+C \omega(1-r),
\end{aligned}
$$

which implies (3.9).

If $u(\rho y) u(r y)<0$, then there exists $r_{0} \in(r, \rho)$ such that $u\left(r_{0} y\right)=0$. It follows that

$$
\begin{aligned}
& |u(\rho y)-u(r y)|=|u(\rho y)|+|u(r y)| \\
& \quad=|u(\rho y)|-|u(y)|+|u(y)|-\left|u\left(r_{0} y\right)\right|+|u(r y)|-|u(y)|+|u(y)|-\left|u\left(r_{0} y\right)\right| \\
& \quad \leq C \omega(1-\rho)+C \omega\left(1-r_{0}\right)+C \omega(1-r)+C \omega\left(1-r_{0}\right) \\
& \quad \leq 4 C \omega(1-r),
\end{aligned}
$$

which completes the proof.

Proof of Theorem 5. It follows from the hypothesis and Lemma 7 that $u$ satisfies (3.9). Then, by Lemmas 5 and $6, u$ satisfies (3.6). Hence $u \in \Lambda_{\omega_{3}}\left(\mathbb{B}_{N}\right)$, by Lemma 3 (with the obvious change of notation) and formula (3.3). This completes the proof.

\section{Proof of Theorem 3}

This time we use Green's formula in the form

$$
\int_{\partial \mathbb{B}_{N}} \frac{1-|x|^{2}}{|x-y|^{N}}\{U(y)-U(x)\} d \sigma(y)=\gamma_{N} \int_{\mathbb{B}_{N}} G_{N}(x, w) \Delta U(w) d \nu(w),
$$

where $U \in C^{2}\left(\mathbb{B}_{N}\right) \cap C\left(\overline{\mathbb{B}}_{N}\right), d \nu$ is the normalized Lebesgue measure on $\mathbb{B}_{N}$, $\gamma_{N}$ a certain constant, and

$$
G_{N}(x, w)=\left\{\begin{array}{l}
\log \left|\frac{1-x \bar{w}}{x-w}\right|, \quad N=2 \quad \text { (complex notation), } \\
|x-w|^{2-N}-\left(1+|x|^{2}|w|^{2}-2\langle x, w\rangle\right)^{1-N / 2}, \quad N \geq 3,
\end{array}\right.
$$

where $\langle\cdot, \cdot\rangle$ denotes the inner product in $\mathbb{R}^{N}$; see, for example, [1, p. 11]. 
Lemma 8. If $f \in H\left(\mathbb{B}_{N}, \mathbb{C}\right)$, then

$$
|\nabla f(0)|^{2} \leq A_{N} \sup _{w \in \mathbb{B}_{N}}\left\{|f(w)|^{2}-|f(0)|^{2}\right\}
$$

where $A_{N}$ is a constant depending only on $N$.

Proof. Assume, as we may, that $U \in H\left(\overline{\mathbb{B}}_{N}, \mathbb{C}\right)$. Taking $U=|f|^{2}$ and using the formula $\Delta\left(|f|^{2}\right)=2|\nabla f|^{2}$, we get from Green's formula

$$
\int_{\partial \mathbb{B}_{N}}\left\{|f(y)|^{2}-|f(0)|^{2}\right\} d \sigma(y)=2 \gamma_{N} \int_{\mathbb{B}_{N}} G_{N}(0, w)|\nabla f(w)|^{2} d \nu(w) .
$$

Hence, using the formulas

$$
\int_{\mathbb{B}_{N}} F d \nu=N \int_{0}^{1} r^{N-1} d r \int_{\partial \mathbb{B}_{N}} F(r y) d \sigma(y)
$$

(integration in polar coordinates) and

$$
|\nabla f(0)|^{2} \leq \int_{\partial \mathbb{B}_{N}}|\nabla f(r y)|^{2} d \sigma(y)
$$

(subharmonicity of $|\nabla f|^{2}$ ), we get

$$
|\nabla f(0)|^{2} \leq A_{N} \int_{\partial \mathbb{B}_{N}}\left\{|f(y)|^{2}-|f(0)|^{2}\right\} d \sigma(y)
$$

The result follows.

Proof of Theorem 3. Applying Lemma 8 to the function

$$
g(w)=f(x+(1-|x|) w),
$$

we get

$$
|\nabla f(x)|^{2}(1-|x|)^{2} \leq A_{N} \sup \left\{|f(w)|^{2}-|f(x)|^{2}:|w-x| \leq 1-|x|\right\} .
$$

From this and the hypothesis $|f|^{2} \in \Lambda_{\omega}\left(\mathbb{B}_{N}\right)$ it follows that

$$
|\nabla f(x)| \leq C \frac{\sqrt{\omega(1-|x|)}}{1-|x|} .
$$

It remains to apply Lemma A. 


\section{Proof of Theorem 4}

Proof Case (a). We use the inequality

$$
\left|f^{\prime}(z)\right| \leq \frac{2}{\varepsilon}\left(\sup _{|w-z|<\varepsilon}|f(w)|-|f(z)|\right), \quad \text { where } \varepsilon=1-|z|,
$$

a consequence of Schwarz's lemma (see [17]). Multiplying this by $|f(z)|^{p-1}$, and using the inequality

$$
|f(z)| \leq \sup _{|w-z|<\varepsilon}|f(w)|
$$

we get

$$
\begin{aligned}
|f(z)|^{p-1}\left|f^{\prime}(z)\right| & \leq \frac{2}{\varepsilon}\left(\sup _{|w-z|<\varepsilon}|f(w)||f(z)|^{p-1}-|f(z)|^{p}\right) \\
& \leq \frac{2}{\varepsilon}\left(\sup _{|w-z|<\varepsilon}|f(w)|^{p}-|f(z)|^{p}\right) .
\end{aligned}
$$

The result follows.

Proof Case (b). In this case we start from the inequality

$$
|\nabla f(x)| \leq \frac{N}{\varepsilon}\left(\sup _{|w-x|<\varepsilon}|f(w)|-|f(x)|\right), \quad \text { where } \varepsilon=1-|x| .
$$

and then proceed in the same way as in the case (a). By translation and dilation, the proof of (5.2) reduces to the case $x=0$ and $|f| \leq 1$ in $\mathbb{B}_{N}$. Then we consider the positive harmonic functions $u_{1}=1+f$ and $u_{2}=1-f$. By Harnack's inequality, we have

$$
\left|\nabla u_{j}(0)\right| \leq N u_{j}(0)
$$

which gives $(5.2)(x=0)$ and concludes the case (b).

Proof Case (c). This case seems to be more subtle. We use the following fact (see [16, Theorem 2] or [18, Theorem 9.3.9]).

Theorem B. Let $F$ be a $C^{2}$-function on $\mathbb{B}_{N}$ satisfying the following condition:

$$
|\Delta F(x)| \leq \frac{K}{\varepsilon^{2}} \sup _{|w-x|<\varepsilon}|F(w)-F(x)|, \quad \text { whenever } 0<\varepsilon \leq 1-|x|,
$$

where $K$ is a constant. Then there is a constant $C=C_{K, N}$ such that

$$
|\nabla F(x)| \leq \frac{C}{\varepsilon} \sup _{|w-x|<\varepsilon}|F(w)-F(x)|, \quad \text { whenever } 0<\varepsilon \leq 1-|x| .
$$


Therefore we only have to verify that the function $F=|f|^{p}, p>2$, satisfies (5.3). As usual, it suffices to consider the case where $x=0$ and $\varepsilon=1$.

Using the formula

$$
\Delta\left(\varphi^{\alpha}\right)=\alpha(\alpha-1) \varphi^{\alpha-2}|\nabla \varphi|^{2}+\alpha \varphi^{\alpha-1} \Delta \varphi
$$

with $\varphi=|f|^{2}, \alpha=p / 2$, we get

$$
\Delta\left(|f|^{p}\right)=\frac{p(p-2)}{4}|f|^{p-4}\left|\nabla\left(|f|^{2}\right)\right|^{2}+p|f|^{p-2}|\nabla f|^{2} .
$$

(Here we have used the formula $\Delta|f|^{2}=2|\nabla f|^{2}$.) Now, if $f=u+i v$, then

$$
\begin{aligned}
\left|\nabla\left(|f|^{2}\right)\right|^{2} & =4|u \nabla u+v \nabla v|^{2} \\
& \leq 4\left(u^{2}+v^{2}\right)\left(|\nabla u|^{2}+|\nabla v|^{2}\right) \\
& =4|f|^{2}|\nabla f|^{2} .
\end{aligned}
$$

Combining these relations we get

$$
0 \leq \Delta\left(|f|^{p}\right) \leq p(p-1)|f|^{p-2}|\nabla f|^{2} \quad(p \geq 2) .
$$

On the other hand, multiplying (4.1) by $|f(0)|^{p-2}$ we get

$$
|f(0)|^{p-2}|\nabla f(0)|^{2} \leq A_{N} \sup _{w \in \mathbb{B}_{N}}\left\{|f(w)|^{p}-|f(0)|^{p}\right\} .
$$

It follows that the function $F=|f|^{p}$ satisfies (5.3) with $K=p(p-1) A_{N}$, which was to be proved.

\section{Remarks}

Remark 1. The implication $|f|^{2} \in \Lambda_{\omega}\left(\mathbb{B}_{N}\right) \Longrightarrow|f| \in \Lambda_{\sqrt{\omega}}\left(\mathbb{B}_{N}\right)$, where $f \in H\left(\mathbb{B}_{N}, \mathbb{C}\right)$, can be extended to

$$
|f|^{p} \in \Lambda_{\omega}\left(\mathbb{B}_{N}\right) \Longrightarrow f \in \Lambda_{\omega^{1 / p}}\left(\mathbb{B}_{N}\right),
$$

where $p \geq 2$. To prove this we use (4.1) to get

$$
|\nabla f(0)|^{p} \leq A_{N}^{p / 2} \sup _{w \in \mathbb{B}_{N}}\left\{|f(w)|^{p}-|f(0)|^{p}\right\}
$$

and then proceed as above. Similarly, using inequalities (5.1) and (5.2), we can prove the following:

If $f$ is holomorphic in $\mathbb{D}$ or real valued and harmonic in $\mathbb{B}_{N}$, and if $p>1$, then there holds the implication

$$
|f|^{p} \in \Lambda_{\omega} \Longrightarrow f \in \Lambda_{\omega^{1 / p}} .
$$


Remark 2. It is proved in [19, Theorem 3] that if $F$ satisfies (5.4), then

$$
|\nabla F(0)|^{q} \leq C_{q, N} \int_{\mathbb{B}_{N}}|\nabla F|^{q} d \nu, \quad 0<q<\infty
$$

This applies to the case $F=|u|^{s}, s \geq 1, u \in H\left(\mathbb{B}_{N}, \mathbb{R}\right)$. It turns out that

$$
\left(|u(0)|^{s-1}|\nabla u(0)|\right)^{q} \leq C \int_{\mathbb{B}_{N}}\left(|u|^{s-1}|\nabla u|\right)^{q} d \nu .
$$

which can be rewritten as

$$
|u(0)|^{p}|\nabla u(0)|^{q} \leq C \int_{\mathbb{B}_{N}}|u|^{p}|\nabla u|^{q} d \nu, \quad \text { where } p, q \geq 0 .
$$

In the case $q=0$ this inequality is a consequence of a result of Hardy and Littlewood [10, Theorem 5] (see [8, 11], where this fact was observed and proved). It is of fundamental importance, e.g., in proving Fefferman and Stein's theorem that the nontangential maximal function is dominated by the radial maximal function (see [8] and [9]).

Acknowledgement. The author wishes to thank his gratitude to professor Konstantin Dyakonov for valuable comments.

\section{References}

[1] Aronszajn, N., Creese, T. M. and Lipkin, L. J.: Polyharmonic functions. Oxford Mathematical Monographs. The Clarendon Press, Oxford University Press, New York, 1983.

[2] BöE, B.: A norm on the holomorphic Besov space. Proc. Amer. Math. Soc. 131 (2003), no. 1, 235-241.

[3] Duren, P. L.: Theory of $H^{p}$ spaces. Pure and Applied Mathematics 38. Academic Press, New York-London, 1970.

[4] Dyakonov, K. M.: Equivalent norms on Lipschitz-type spaces of holomorphic functions. Acta Math. 178 (1997), 143-167.

[5] Dyakonov, K. M.: Holomorphic functions and quasiconformal mappings with smooth moduli. Adv. Math. 187 (2004), 146-172.

[6] Dyakonov, K. M.: Strong Hardy-Littlewood theorems for analytic functions and mappings of finite distortion. Math. Z. 249 (2005), no. 3, 597-611.

[7] Dyakonov, K. M.: Addendum to "Strong Hardy-Littlewood theorems for analytic functions and mappings of finite distortion". Math. Z. 254 (2006), no. $2,433-437$.

[8] Fefferman, C. And Stein, E. M.: $H^{p}$ spaces of several variables. Acta Math. 129 (1972), 137-193. 
[9] Garnett, J. B.: Bounded analytic functions. Pure and Applied Mathematics, 96. Academic Press, Inc., New York-London, 1981.

[10] Hardy, G. H. and Littlewood, J. E.: Some properties of conjugate functions. J. Reine Angew. Math. 167 (1932), 405-423.

[11] Kuran, Ü.: Subharmonic behaviour of $h^{p}$ ( $p>0, h$ harmonic). J. London Math. Soc. (2) 8 (1974), 529-538.

[12] Nolder, C. A. And Oberlin, D. M.: Moduli of continuity and a HardyLittlewood theorem. In Complex Analysis, Joensuu 1987, 265-272. Lecture Notes in Math. 1351. Springer, Berlin, 1988.

[13] Pavlović, M.: Lipschitz spaces and spaces of harmonic functions in the unit disc. Michigan Math. J. 35 (1988), no. 2, 301-311.

[14] Pavlović, M.: On the moduli of continuity of $H^{p}$-functions with $0<p<$ 1,. Proc. Edinburgh Math. Soc. (2) 35 (1992), 89-100.

[15] Pavlović, M.: On subharmonic behaviour and oscillation of functions on balls in $\mathbb{R}^{n}$. Publ. Inst. Math. (Beograd) 55 (1994), 18-22.

[16] Pavlović, M.: Subharmonic behaviour of smooth functions. Mat. Vesnik 48 (1996), 15-21.

[17] PAVlović, M.: On K.M. Dyakonov's paper: "Equivalent norms on Lipschitz-type spaces of holomorphic functions". Acta Math. 183 (1999), 141-143.

[18] Pavlović, M.: Introduction to function spaces on the disk. Posebna Izdanja 20. Matematički Institut SANU, Belgrade, 2004.

[19] Pavlović, M.: Remarks on $L^{p}$-oscillation of the modulus of a holomorphic function. J. Math. Anal. Appl. 326 (2007), no. 1, 1-11.

[20] Rudin, W.: Function theory in the unit ball of $\mathbb{C}^{n}$. Grundlehren der Mathematischen Wissenschaften 241. Springer-Verlag, New York-Berlin, 1980.

[21] Stein, E. M.: Singular integrals and differentiability properties of functions. Princeton Mathematical Series 30. Princeton University Press, Princeton, N.J. 1970.

Recibido: 26 de octubre de 2005

Revisado: 1 de junio de 2006

Miroslav Pavlović

Matematički fakultet

Studentski trg 16

11001 Beograd, p.p. 550, Serbia

pavlovic@matf.bg.ac .yu

The author is supported by MNZŽS Grant No. ON144010, Serbia. 